\title{
Assessment of common extensor tendon elasticity in patients with lateral epicondylitis using shear wave elastography
}

\author{
Bihui Zhu, Yingqi You, Xi Xiang, Liyun Wang, Li Qiu \\ Department of Ultrasound, West China Hospital of Sichuan University, Chengdu 610041, China \\ Correspondence to: Li Qiu. Department of Ultrasound, West China Hospital of Sichuan University, No.37 Guo Xue Xiang, Chengdu 610041, China. \\ Email: wsqiuli@126.com.
}

\begin{abstract}
Background: To investigate the role of shear wave elastography (SWE) in patients with lateral epicondylitis (LE) by assessing the common extensor tendon (CET) elasticity.

Methods: A total of 62 unilateral LE patients were enrolled. Shear wave speed (SWS) and the thickness of CET in both elbows, along with the involved elbows with pre- and post-treatment, were obtained by SWE. The differences between groups, inter- and intra-observer agreements, and diagnostic accuracy were analyzed with a paired $t$-test, intraclass correlation coefficients (ICCs), and receiver operator characteristic (ROC) curve, respectively.

Results: LE patients had significantly lower SWS on lesion sides compared to healthy elbows $(\mathrm{P}<0.05)$. The SWS of involved elbows were significantly higher after non-operation treatment than before treatment. The inter- and intra-observer agreements were excellent (ICCs: 0.900-0.993) for SWE measurements. Moreover, a $12.2 \mathrm{~m} / \mathrm{s}$ cutoff value of mean SWS $\left(C_{\text {mean }}\right)$ for discriminating LE patients from healthy subjects revealed a sensitivity and specificity of $93 \%$ and $93 \%$, respectively.

Conclusions: SWE is a valid imaging technique for the diagnosis of LE and monitoring of the treatment effect. Future studies are essential for investigating the correlations among clinical examinations, conventional ultrasound, and SWE.
\end{abstract}

Keywords: Common extensor tendon (CET); elasticity; lateral epicondylitis (LE); shear wave speed (SWS); elastography

Submitted Jun 20, 2019. Accepted for publication Oct 09, 2019.

doi: 10.21037/qims.2019.10.07

View this article at: http://dx.doi.org/10.21037/qims.2019.10.07

\section{Introduction}

Lateral epicondylitis (LE), known as "tennis elbow", is the most common cause of lateral elbow pain due to the overuse of the common extensor tendon (CET), which has a prevalence of $1-3 \%$ in the general population (1-5). The diagnosis of LE is based primarily on clinical examination, like visual analogy scale (VAS), and often confirmed with diagnostic imaging including conventional ultrasound (US), magnetic resonance imaging (MRI), real-time sonoelastography, etc. for cases of atypical presentation or those resistant to conservative treatment $(6,7)$. Furthermore, imaging techniques provide the information related to the physiopathology of disease condition and determine the radiological severity of $\mathrm{LE}$, and can be essential in assessing the level of impairment, planning treatment strategies, or guiding surgery $(5,8,9)$. Although MRI has been widely considered as a reliable complementary method for LE assessment, the high cost, lengthy examination time, and several contraindications may limit its widespread use (6). Currently, the US is increasingly playing a vital role in the diagnosis of LE with inexpensive, non-invasive, and dynamic features. US findings in the CET for LE are characterized by edema, decreased echogenicity, epicondylar cortical irregularity or spur formation, the severity of the disease with evidence of tendon thickening, and Doppler 


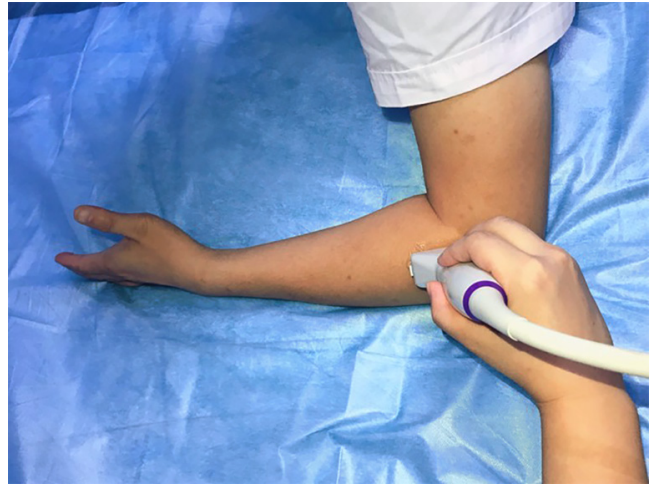

Figure 1 Examples of common extensor tendon (CET) measurements using shear wave elastography (SWE). The patient sat in front of the examiner with the elbow flexed at $90^{\circ}$ and the thumb up.

activity (10-12). However, the potential role of the US is limited because it has been reported that both its sensitivity (US: $64 \%$ to $88 \%$, MRI: $90 \%$ to $100 \%$ ) and specificity (US: $36 \%$ to $48.5 \%$, MRI: $67 \%$ to $89 \%$ ) are lower than those of MRI (13-15).

Recent studies (16-19) in which elastography was used to evaluate the mechanical properties of tendons have reported that elastographic methods may detect the changes in tendon stiffness qualitatively and quantitatively, as well as monitor and guide ongoing treatments of tendinopathy. Shear wave elastography (SWE) is one of the main elastographic techniques, which can be induced through various methods. One such method works on the basis of multiple focused push beams (supersonic shear imaging) generating shear waves and ultra-high frame rate ultrasonic imaging of the resulting shear wave propagation to calculate the shear wave speed (SWS) at different depths within the tissue $(19,20)$.

Previous studies $(21,22)$ have applied SWE to assess the Achilles tendon and have demonstrated that SWE is valuable in quantitatively measuring the biomechanical properties of the Achilles tendon in vivo. However, SWE of the CET in LE has seldom been investigated, and the majority of studies only evaluated the CET with real-time sonoelastography $(23,24)$. Therefore, in order to determine whether the SWE can be used in the diagnosis of LE, the present study assessed CET elasticity in patients with unilateral LE by quantitative SWE and monitored the disease condition before and after the non-operative therapy

\section{Methods}

This study was performed with the approval of the West China Hospital of Sichuan University Ethics Committee and written informed consent was obtained from each participant. There were 62 patients assessed by the orthopedic surgeon with a definitive clinical diagnosis of unilateral LE enrolled in this study from June 2018 to December 2018. The criteria for the diagnosis of LE included patients with a typical history, tenderness on the lateral epicondyle, and pain aggravated by extension and twist that radiated from the lateral forearm to the palm. Both elbows of all patients were examined by SWE before and after non-operative treatment. All of the patients received physical therapy for one month after the first evaluation. Participants were not included if they presented with a known history of bilateral LE, elbow fracture, elbow surgery, other systemic musculoskeletal disorders, or pregnancy. General information such as sex, age, dominant hand, and disease duration were recorded.

Elastography and thickness examinations were performed with an Aixplorer US system (SuperSonic Imagine, Aixplorer, Aixen-Provence, France) with an SL 4-15 MHz linear array probe (SuperSonic Imagine). Each patient was asked to sit in front of the examiner with the elbow flexed at $90^{\circ}$ and the thumb up, and a relaxing position of the forearm was maintained during the measurement (Figure 1). The transducer was placed perpendicularly to the lateral epicondyle onto the skin surface with light contact using a coupling agent so as to avoid the compression effect $(5,25)$. The CET attaching to the lateral epicondyle of the humerus was scanned in a longitudinal plane (a parallel orientation to CET fibers) for obtaining the standardized images, and the elastograms appeared as an overlay in dualmode alongside B-mode image. The thickness of the CET was defined as the distance between the surface of the extensor tendon and the lateral epicondylar cortex. The color code indicated the tissue elasticity within the regions of interest (ROI) ranging from blue to red, representing soft to hard respectively. Then, the integrated SWE elastograms automatically displayed SWS and Young's modulus, and SWS was the primary measurement used for the analysis. A superficial musculoskeletal setting was chosen, and the scale was adjusted to $600 \mathrm{kPa}$ to acquire optimal and standardized images. The size of the ROI was fixed to $2 \mathrm{~mm}$ in all cases. Care was taken not to place the circular ROI outside the CET and close to bone. Figure 2 represents the 


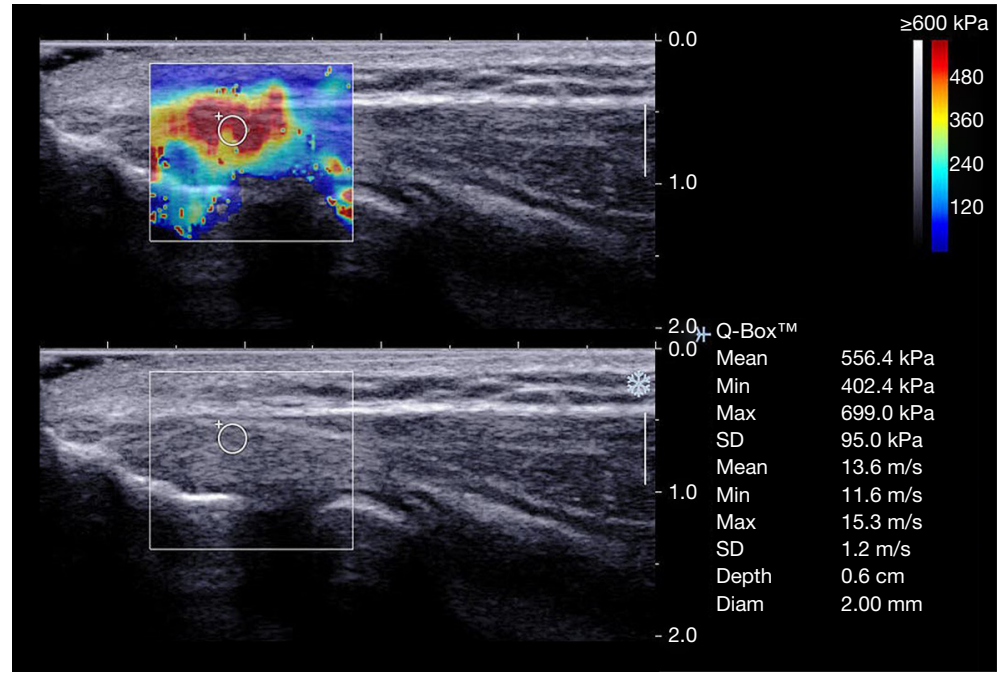

Figure 2 Shear wave elastography (SWE) image of the healthy common extensor tendon (CET) on longitudinal axis in a 36-year-old female patient with unilateral lateral epicondylitis (LE). The bottom image is a longitudinal gray-scale ultrasound (US) scan of CET with superimposed color box borders for the SWE map, while there is a circular ROI for quantitative measurements on the top image. Quantitative SWE measurements show the $C_{\text {mean }}$ is $13.6 \mathrm{~m} / \mathrm{s}$.

standardized SWE image.

Two independent examiners conducted the elastographic measurements. An experienced operator 1 (10-year experience of musculoskeletal US) examined all 62 subjects. Operator 2 (5-year experience of musculoskeletal US) examined 20 subjects randomly chosen for the inter-observer reliability. Within 1 week after the first examination, the same subjects were rechecked by operator 1 to assess intra-observer reliability. The two examiners were blinded to the results assessed by each other. After the non-operative treatment, operator 1 performed an elastographic evaluation of all patients again as previously described. Three separate SWE and thickness measurements were carried out from approximately the same area of the tendon, and the average mean, minimum, and maximum SWS $(\mathrm{m} / \mathrm{s})$ within each ROI were used for analysis, in addition to the average thickness.

Statistical analyses were performed using SPSS software (Version 20.0, IBM, NY, USA). Continuous variables were expressed as mean \pm standard deviation. The KolmogorovSmirnov test was used to check for normal distribution data (Figure S1). The SWS and thickness of both the bilateral elbow and the lesion side before and after nonoperative therapy were determined using the paired $t$-test. Pearson correlation was used in the correlation analysis between the demographic data and SWS. The intra- and inter-observer agreements were assessed with intraclass correlation coefficients (ICCs). The ICCs between 0.75 and 0.9 , and those greater than 0.90 were indicative of good and excellent reliability, respectively (26). Moreover, SWS of the CET was used for receiver operator characteristic (ROC) curve analysis. Cutoff values of mean, minimum, and maximum velocities $\left(C_{\text {mean }}, C_{\min }\right.$, and $\left.C_{\max }\right)$ were chosen by maximizing the Youden index on the estimated curves. The sensitivity, specificity, and accuracy in the diagnosis of LE on the basis of SWE measurements were calculated. $\mathrm{P}<0.05$ was considered a statistically significant difference.

\section{Results}

A total of 62 patients with unilateral LE were included, and the baseline demographic characteristics of the study participants are summarized in Table 1 .

The thickness in unilateral LE patients revealed a significantly higher value in affected CET $(5.31 \pm 0.49 \mathrm{~mm})$ than in healthy CET $(3.95 \pm 0.37 \mathrm{~mm})(\mathrm{P}<0.05)$. In addition, a statistical difference in thickness of CET was detected between measures before and after non-operation therapy $(\mathrm{P}<0.05)$, and the thickness decreased after non-operation therapy $(4.19 \pm 0.35 \mathrm{~mm})$.

In healthy elbows, the elastograms demonstrated stiff tendon structures corresponding mostly to the red region, and the most blue or green areas were observed in elbows with LE (Figures 3,4). Moreover, the side-to-side analysis 
Table 1 The baseline demographic data of the patients

\begin{tabular}{lc}
\hline Characteristic & Patients $(\mathrm{n}=62)$ \\
\hline Age [range], years & $44.1 \pm 4.3[30-50]$ \\
Sex (female/male) & $39 / 23$ \\
Hand dominance (left/right) & $8 / 54$ \\
Involvement (left/right) & $11 / 51$ \\
Disease duration [range], days & $52.1 \pm 40.2[1-180]$ \\
\hline
\end{tabular}

revealed a significant difference in terms of CET elasticity in the elbows with LE and in healthy control elbows, in which three velocities on the involvement side were significantly lower than those on the healthy side $(\mathrm{P}<0.05)$. Additionally, the SWS obtained in the elbows with LE showed significantly higher values in post-therapy than in pre-therapy $(\mathrm{P}<0.05)($ Table 2$)$.

The mean SWS was not associated with age and gender $(\mathrm{P}=0.363$ and 0.192 , respectively), and there
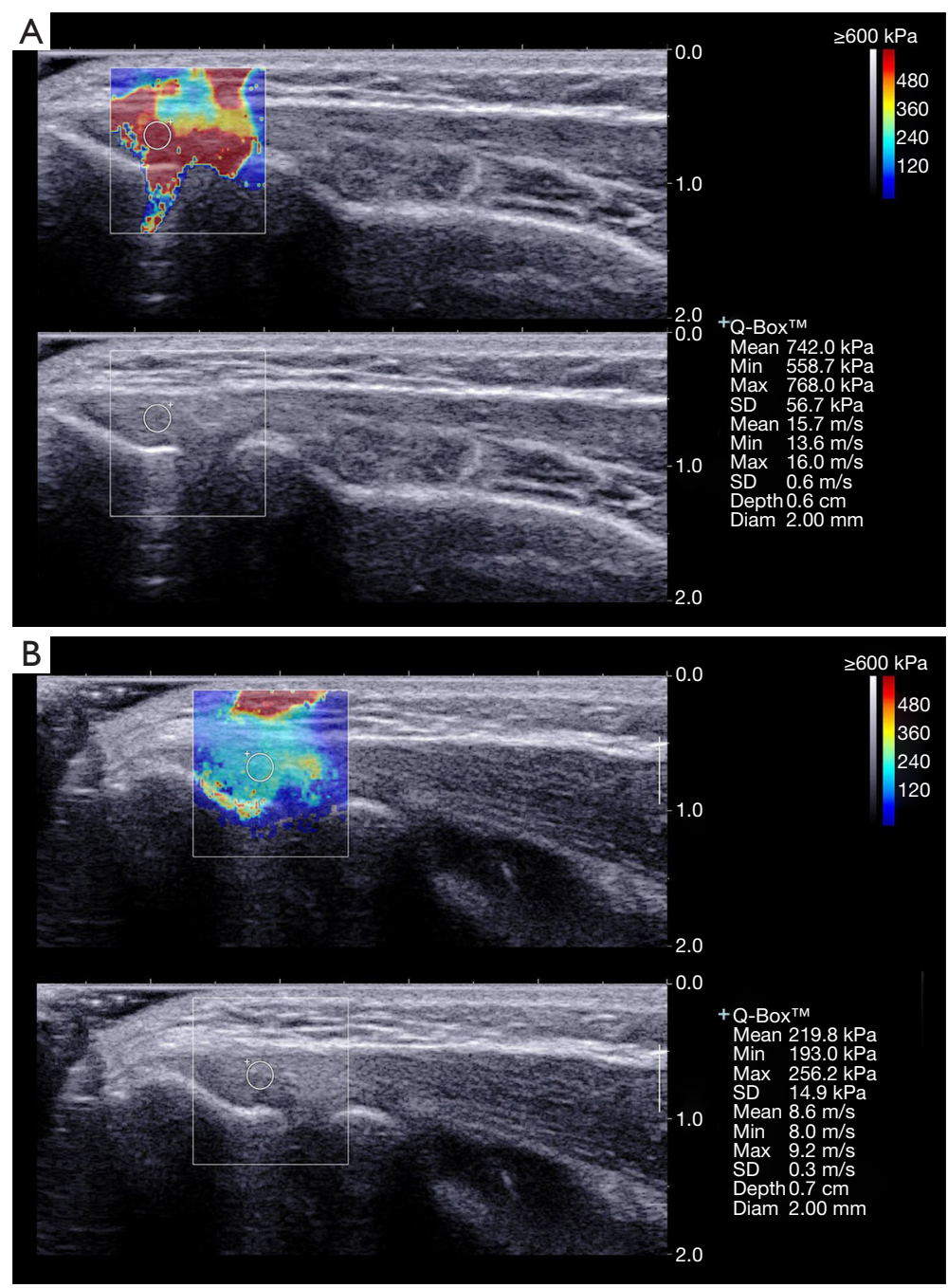

Figure 3 Shear wave elastography (SWE) images of the bilateral common extensor tendon (CET) on longitudinal axis in a 31-year-old female patient. (A) SWE of the healthy CET showing a mostly red region indicating the stiff tendon structures. Quantitative SWE measurements show the $C_{\text {mean }}$ is $15.7 \mathrm{~m} / \mathrm{s}$. (B) SWE of involved CET showing the most blue or green areas indicating the soft tendon structures. Quantitative SWE measurements show the $C_{\text {mean }}$ is $8.6 \mathrm{~m} / \mathrm{s}$. 

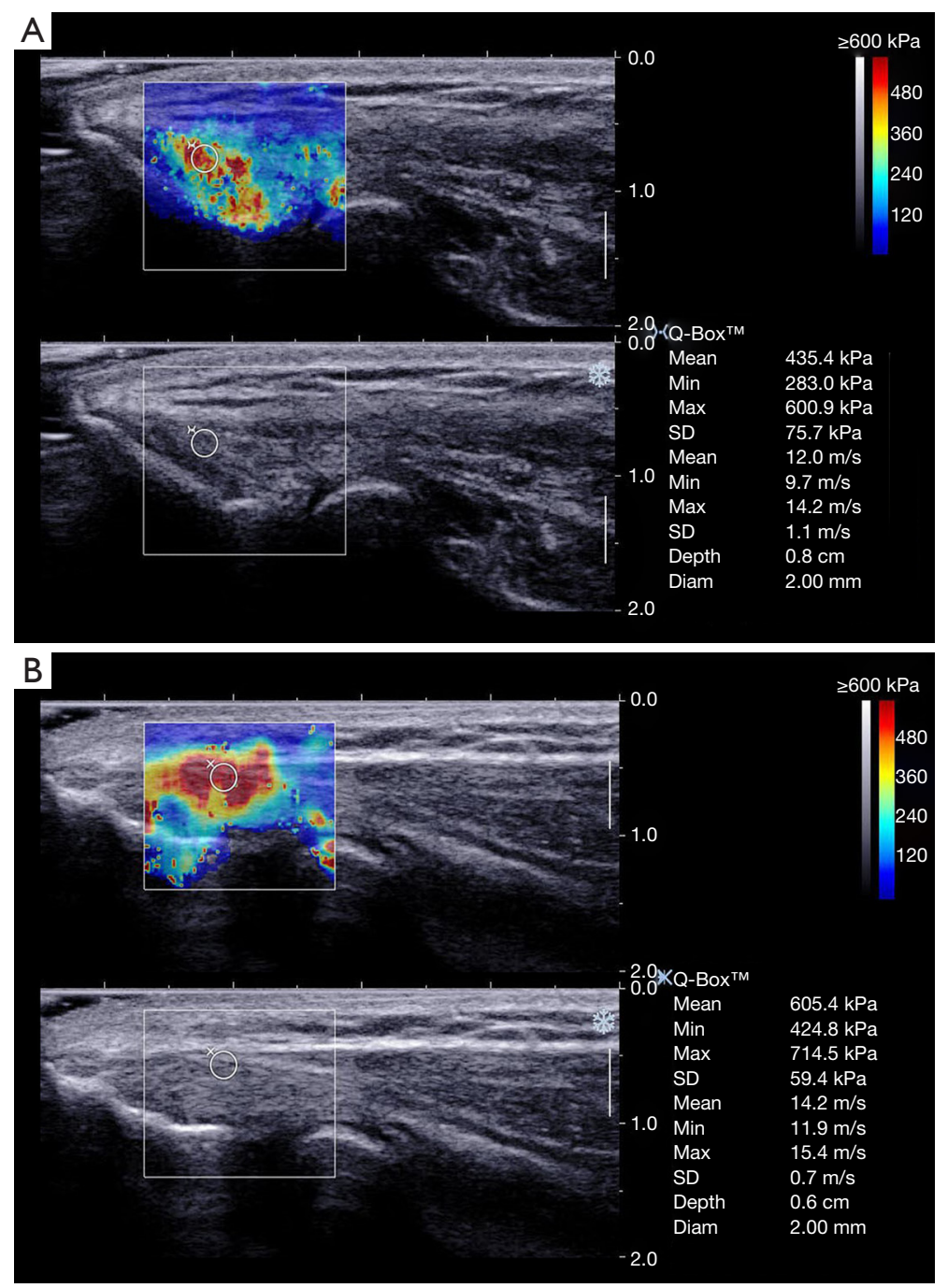

Figure 4 Shear wave elastography (SWE) images of the same common extensor tendon (CET) with LE on longitudinal axis (A) before non-operative treatment and (B) after non-operative treatment in a 40-year-old male patient. The change of shear wave speed (SWS) demonstrated that SWE could monitor the therapeutic effect.

Table 2 The SWS in bilateral elbows and involvement elbows for pre- and post-therapy

\begin{tabular}{|c|c|c|c|c|c|c|}
\hline SWS (m/s) & \multicolumn{3}{|c|}{ Bilateral elbows } & \multicolumn{3}{|c|}{ LE elbows } \\
\hline$C_{\text {mean }}$ & $9.6 \pm 1.4$ & $13.6 \pm 1.1$ & $<0.05$ & $9.6 \pm 1.4$ & $12.1 \pm 1.7$ & $<0.05$ \\
\hline$C_{\min }$ & $8.3 \pm 1.1$ & $11.1 \pm 2.0$ & $<0.05$ & $8.3 \pm 1.1$ & $9.60 \pm 1.9$ & $<0.05$ \\
\hline$C_{\max }$ & $10.9 \pm 1.8$ & $15.1 \pm 0.8$ & $<0.05$ & $10.9 \pm 1.8$ & $13.9 \pm 1.1$ & $<0.05$ \\
\hline
\end{tabular}

Data are mean \pm standard deviation. SWS, shear wave speed; LE, lateral epicondylitis; $C_{\text {mean }}$, mean velocities; $C_{\text {min }}$, minimum velocities; $C_{\max }$, maximum velocities. 
Table 3 ICCs for inter- and intra-observer reliability for SWE measurements

\begin{tabular}{|c|c|c|c|c|c|}
\hline Parameter & \multicolumn{3}{|c|}{ SWS (m/s) } & \multicolumn{2}{|c|}{ ICCs } \\
\hline$C_{\text {mean }}$ & $11.1 \pm 2.1$ & $11.1 \pm 2.0$ & $11.3 \pm 2.2$ & $0.993(0.983-0.997)$ & $0.986(0.964-0.994)$ \\
\hline$C_{\text {min }}$ & $8.2 \pm 1.1$ & $8.0 \pm 1.1$ & $8.1 \pm 1.3$ & $0.961(0.903-0.985)$ & $0.900(0.746-0.960)$ \\
\hline$C_{\max }$ & $9.8 \pm 1.6$ & $9.8 \pm 1.6$ & $9.7 \pm 1.7$ & $0.979(0.946-0.992)$ & $0.971(0.927-0.989)$ \\
\hline
\end{tabular}

Data are means \pm standard deviation or median $(95 \% \mathrm{Cl})$. SWS, shear wave speed; $C_{\text {mean }}$, mean velocities; $C_{\min }$, minimum velocities; $C_{\text {max }}$, maximum velocities; ICCs, intraclass correlation coefficients; $\mathrm{Cl}$, confidence interval.

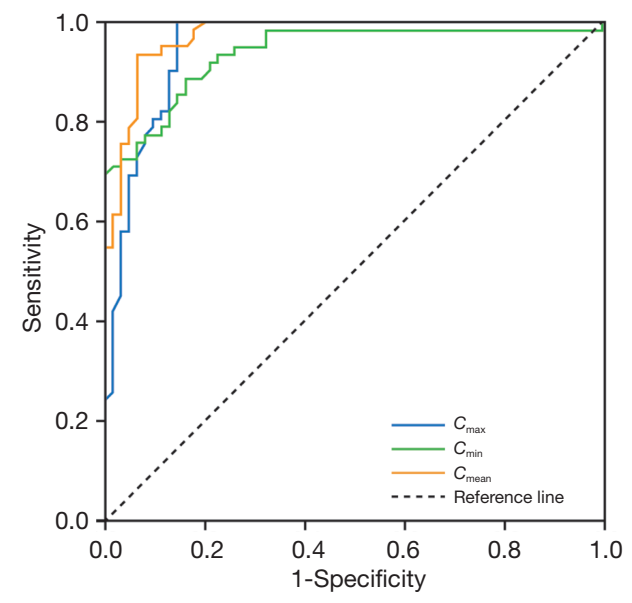

Figure 5 The receiver operator characteristic (ROC) curve of mean, minimum, and maximum velocities $\left(C_{\text {mean }}, C_{\min }\right.$ and $\left.C_{\max }\right)$ from patients with lateral epicondylitis (LE).

was a low correlation between duration and mean SWS $(\mathrm{P}<0.05, \mathrm{R}=0.258)$. However, an excellent correlation was obtained between dominance hand and involvement elbow $(\mathrm{P}<0.001, \mathrm{R}=0.829)$. The results of inter- and intra-observer reliability of SWE measurements in 20 patients are presented in Table 3 and Figure S2. There were excellent inter- and intra-observer agreements, as ICCs ranged from 0.900 to 0.993 .

The ROC curves for the diagnosis of LE based on the SWS are illustrated in Figure 5, in which the $C_{\text {mean }}$ displays the highest diagnostic accuracy. When the $C_{\text {mean }}$ was used, both the sensitivity (93\%) and specificity (93\%) were high. The mean SWS cutoff value for determining the diagnosis of LE was $12.2 \mathrm{~m} / \mathrm{s}$, with an area under the curve of 0.973 (95\% CI: 0.949-0.997) (Table 4).

\section{Discussion}

LE is more common in individuals $40-45$ years of age
Table 4 Sensitivity, specificity, and AUC values according to cutoff values

\begin{tabular}{|c|c|c|c|}
\hline $\begin{array}{l}\text { Parameter and } \\
\text { cutoff value }\end{array}$ & Sensitivity & Specificity & AUC $(95 \% \mathrm{Cl})$ \\
\hline$C_{\text {mean }}, 12.2 \mathrm{~m} / \mathrm{s}$ & $93 \%$ & $93 \%$ & $0.973(0.949-0.997)$ \\
\hline$C_{\min }, 9.27 \mathrm{~m} / \mathrm{s}$ & $89 \%$ & $84 \%$ & $0.939(0.897-0.981)$ \\
\hline$C_{\max }, 12.9 \mathrm{~m} / \mathrm{s}$ & $100 \%$ & $85 \%$ & $0.952(0.915-0.989)$ \\
\hline
\end{tabular}

or people with manual labor occupations, with men and women being affected equally $(27,28)$. Despite tennis players making up less than $10 \%$ of the patient population, half of the tennis players do develop pain around the elbow, $75 \%$ of which is characteristic of true "tennis elbow" (29,30). The diagnosis is usually clinical, but some LE patients may benefit from additional imaging for a specific differential diagnosis. Nowadays, alternative imaging is needed to help complementary diagnosis because of some limits of US and MRI, of which elastography is gradually attracting public attention. Increased tendon compressibility, indicative of tendon softening, is considered as a new sonographic sign of common extensor tendinopathy (31). Therefore, evaluation of tendon softening by elastography may be a beneficial method for discrimination of patients with LE.

Lee et al. (13) measured the thickness of CET in patients with LE and found it was significantly thicker than that of healthy control subjects, which is consistent with our results. This phenomenon may be ascribed to the swelling caused by pathological changes of CET in LE, which leads to an increase in thickness. However, many dense collagen fibers contributing to increasing the thickness of normal CET probably results in stiffer elasticity (32).

Our study has demonstrated that the CET is softer in a diseased elbow when measured with SWE. Şendur et al. (32) 
found that there was no difference of the CET elasticity in healthy volunteers between bilateral elbows. The CET origin is made up of the extensor carpi radialis longus and brevis, extensor digitorum communis, extensor digiti minimi, and the extensor carpi ulnaris (30). Microtears may occur when the tendon experiences excessive stress which will lead to the degenerative changes within the tendon, namely tendinosis. LE, reflecting an overuse injury primarily due to repetitive strain from tasks and activities, is a degenerative process and is characterized of angiofibroblastic hyperplasia, micro-rupture, an abundance of fibroblasts, vascular hyperplasia, and notably, a lack of traditional inflammatory cells within the tissue $(15,30,33)$. These changes have been shown to play the most critical pathophysiological role in the production of LE and make the tendon softer. A decrease in CET stiffness was previously reported in patients with LE by real-time sonoelastography $(23,24)$, which is consistent with our results wherein SWE-based measurements of the CET could directly reflect the change associated with the effect of LE on the tendon. Moreover, Sahan et al. (17) found that there was a statistically significant difference in terms of elasticity between the long head of the biceps tendon (LHBT) tendinosis and control groups with SWE (LOGIQ E9 sonographic system, GE Healthcare) and concluded that SWE may be a useful diagnostic tool for LHBT tendinosis. Therefore, SWE may help to discriminate a healthy elbow from a LE elbow, and with the diagnosis of LE.

Another finding in our study was that the SWS was significantly higher in patients after non-operative management than before it. This may be ascribed to the individual difference in treatment effect and short treatment duration so that the post-therapy SWS did not increase to healthy levels. In fact, imaging is not necessary for LE unless the patient is not responding to nonoperative management modalities. However, our result demonstrated that SWE could not only help the diagnosis of the LE but also monitor the effectiveness of treatment.

The intra- and inter-observer agreements were assessed in our study. The measurements with SWE for CET obtained excellent ICCs (values of ICCs ranged from 0.900 to 0.993 for $C_{\text {mean }}, C_{\min }$, and $C_{\max }$ ). The good intra- and inter-observer reliability indicates that this technique is reproducible for the evaluation of CET and seldom dependent on operators. Sendur et al. (32) applied SWE to evaluate the normal CET stiffness and showed the same significant interobserver agreement. However, they proposed that the results of the two observers were nearly equal because of the utilization of the same technique and learning methods, that may be why we achieved excellent ICCs. Therefore, the standard operating method makes SWE reliable and feasible to measure the LE.

The $C_{\text {mean }}$ with a threshold of $12.2 \mathrm{~m} / \mathrm{s}$ showed the best area under curve among mean, minimum, and maximum SWS, with a sensitivity and specificity of $93 \%$ and $93 \%$, respectively. In the reported literature, both the sensitivity and specificity in the diagnosis of LE are better for SWE than for the gray-scale US (13-15). This finding implies it could favorably distinguish patients with LE from healthy subjects by using SWE, and the mean SWS could be used to identify LE better.

Our study has several limitations. First, the previous studies measured the three sections of CET (23), but we only chose one location where the lesion was. Another limitation was the small number of participants. In addition, we did not explore the correlations between SWE and clinical examinations, or the conventional US. We performed measurements only concerning SWE, and future studies would be essential to investigate the comparison among these different evaluation methods.

In this study, the SWS of CET was lower under SWE in LE elbows compared to healthy elbows, and it increased after treatment. The excellent intra- and inter-observer reliability indicates that SWE is a reproducible technique for the evaluation of CET. Moreover, the sensitivity and specificity for SWE were both good in the diagnosis of LE. In conclusion, SWE with high reproducibility and diagnostic accuracy could become a valid imaging method for LE and could help monitor the therapeutic effect.

\section{Acknowledgments}

Funding: This study was supported by the National Natural Science Foundation of China (81671696) and Sichuan Science and Technology Program (2018RZ0086, 2019YFS0219).

\section{Footnote}

Conflicts of Interest: The authors have no conflicts of interest to declare.

Ethical Statement: The experimental protocols were approved by the West China Hospital of Sichuan University Ethics Committee. Informed consent was obtained from each participant for the procedures. 


\section{References}

1. Gündüz R, Malas FU, Borman P, Kocaoglu S, Ozcakar L. Physical therapy, corticosteroid injection, and extracorporeal shock wave treatment in lateral epicondylitis. Clinical and ultrasonographical comparison. Clin Rheumatol 2012;31:807-12.

2. Shillito M, Soong M, Martin N. Radiographic and Clinical Analysis of Lateral Epicondylitis. J Hand Surg Am 2017;42:436-42.

3. Latham SK, Smith TO. The diagnostic test accuracy of ultrasound for the detection of lateral epicondylitis: a systematic review and meta-analysis. Orthop Traumatol Surg Res 2014;100:281-6.

4. Zeisig E. Natural course in tennis elbow--lateral epicondylitis after all? Knee Surg Sports Traumatol Arthrosc 2012;20:2549-52.

5. Toprak U, Baskan B, Ustuner E, Oten E, Altin L, Karademir MA, Bodur H. Common extensor tendon thickness measurements at the radiocapitellar region in diagnosis of lateral elbow tendinopathy. Diagn Interv Radiol 2012;18:566-70.

6. Bachta A, Rowicki K, Kisiel B, Zabicka M, ElertKopec S, Plominski J, Tlustochowicz W, Maliborski A. Ultrasonography versus magnetic resonance imaging in detecting and grading common extensor tendon tear in chronic lateral epicondylitis. PLoS One 2017;12:e0181828.

7. Kotnis NA, Chiavaras MM, Harish S. Lateral epicondylitis and beyond: imaging of lateral elbow pain with clinicalradiologic correlation. Skeletal Radiol 2012;41:369-86.

8. Chelly JE. Usefulness of Ultrasound in Refractory Lateral Epicondylitis. Pain Med 2017;18:395.

9. Walton MJ, Mackie K, Fallon M, Butler R, Breidahl W, Zheng MH, Wang A. The reliability and validity of magnetic resonance imaging in the assessment of chronic lateral epicondylitis. J Hand Surg Am 2011;36:475-9.

10. Krogh TP, Fredberg U, Christensen R, StengaardPedersen K, Ellingsen T. Ultrasonographic assessment of tendon thickness, Doppler activity and bony spurs of the elbow in patients with lateral epicondylitis and healthy subjects: a reliability and agreement study. Ultraschall Med 2013;34:468-74.

11. Ural FG, Ozturk GT, Boluk H, Akkus S. Ultrasonographic Evaluation of Acupuncture Effect on Common Extensor Tendon Thickness in Patients with Lateral Epicondylitis: A Randomized Controlled Study. J Altern Complement Med 2017;23:819-22.

12. Gürçay E, Karaahmet OZ, Kara M, Onat SS, Ata AM,
Unlu E, Ozcakar L. Ultrasonographic Evaluation of the Radial Nerves in Patients with Unilateral Refractory Lateral Epicondylitis. Pain Med 2017;18:396-402.

13. Lee MH, Cha JG, Jin W, Kim BS, Park JS, Lee HK, Hong HS. Utility of sonographic measurement of the common tensor tendon in patients with lateral epicondylitis. AJR Am J Roentgenol 2011;196:1363-7.

14. Obradov M, Anderson PG. Ultra sonographic findings for chronic lateral epicondylitis. Jbr-btr 2012;95:66-70.

15. Faro F, Wolf JM. Lateral epicondylitis: review and current concepts. J Hand Surg Am 2007;32:1271-9.

16. Gulledge CM, Baumer TG, Juliano L, Sweeney M, McGinnis M, Sherwood A, Moutzouros V, Bey MJ. Shear wave elastography of the healing human patellar tendon following ACL reconstruction. Knee 2019;26:347-54.

17. Sahan MH, Inal M, Burulday V, Kultur T. Evaluation of tendinosis of the long head of the biceps tendon by strain and shear wave elastography. Med Ultrason 2018;20:192-8.

18. Park GY, Kwon DR. Application of real-time sonoelastography in musculoskeletal diseases related to physical medicine and rehabilitation. Am J Phys Med Rehabil 2011;90:875-86.

19. Prado-Costa R, Rebelo J, Monteiro-Barroso J, Preto AS. Ultrasound elastography: compression elastography and shear-wave elastography in the assessment of tendon injury. Insights Imaging 2018;9:791-814.

20. Ahmadzadeh SMH, Chen X, Hagemann H, Tang MX, Bull AMJ. Developing and using fast shear wave elastography to quantify physiologically-relevant tendon forces. Med Eng Phys 2019;69:116-22.

21. Payne C, Watt P, Cercignani M, Webborn N. Reproducibility of shear wave elastography measuresof the Achilles tendon. Skeletal Radiol 2018;47:779-84.

22. Zhang L, Yong Q, Pu T, Zheng C, Wang M, Shi S, Li L. Grayscale ultrasonic and shear wave elastographic characteristics of the Achilles' tendon in patients with familial hypercholesterolemia: A pilot study. Eur J Radiol 2018;109:1-7.

23. De Zordo T, Lill SR, Fink C, Feuchtner GM, Jaschke W, Bellmann-Weiler R, Klauser AS. Real-Time Sonoelastography of Lateral Epicondylitis: Comparison of Findings Between Patients and Healthy Volunteers. AJR Am J Roentgenol 2009;193:180-5.

24. Kocyigit F, Kuyucu E, Kocyigit A, Herek DT, Savkin R, Aslan UB, Karabulut N. Association of real-time sonoelastography findings with clinical parameters in lateral epicondylitis. Rheumatol Int 2016;36:91-100. 
25. Itoigawa Y, Maruyama Y, Kawasaki T, Wada T, Yoshida K, An KN, Kaneko K. Shear Wave Elastography Can Predict Passive Stiffness of Supraspinatus Musculotendinous Unit During Arthroscopic Rotator Cuff Repair for Presurgical Planning. Arthroscopy 2018;34:2276-84.

26. Koo TK, Li MY. A Guideline of Selecting and Reporting Intraclass Correlation Coefficients for Reliability Research. J Chiropr Med 2016;15:155-63.

27. Chevinsky JD, Newman JM, Shah NV, Pancholi N, Holliman J, Sodhi N, Eldib A, Naziri Q, Zikria BA, Reilly JP, Barbash SE, Urban WP. Trends and Epidemiology of Tennis-Related Sprains/Strains in the United States, 2010 to 2016. Surg Technol Int 2017;31:333-8.

28. Kwapisz A, Prabhakar S, Compagnoni R, Sibilska A, Randelli P. Platelet-Rich Plasma for Elbow Pathologies: a Descriptive Review of Current Literature. Curr Rev

Cite this article as: Zhu B, You Y, Xiang X, Wang L, Qiu L. Assessment of common extensor tendon elasticity in patients with lateral epicondylitis using shear wave elastography. Quant Imaging Med Surg 2020;10(1):211-219. doi: 10.21037/ qims.2019.10.07
Musculoskelet Med 2018;11:598-606.

29. Buchanan BK, Varacallo M. Tennis Elbow (Lateral Epicondylitis). StatPearls. Treasure Island (FL), 2019.

30. Tosti R, Jennings J, Sewards JM. Lateral epicondylitis of the elbow. Am J Med 2013;126:357.e1-6.

31. Khoury V, Cardinal E. "Tenomalacia": a new sonographic sign of tendinopathy? Eur Radiol 2009;19:144-6.

32. Şendur HN, Cindil E, Cerit M, Demir NB, Sendur $\mathrm{AB}$, Oktar SO. Interobserver variability and stiffness measurements of normal common extensor tendon in healthy volunteers using shear wave elastography. Skeletal Radiol 2019;48:137-41.

33. Vaquero-Picado A, Barco R, Antuna SA. Lateral epicondylitis of the elbow. EFORT Open Rev 2017;1:391-7. 

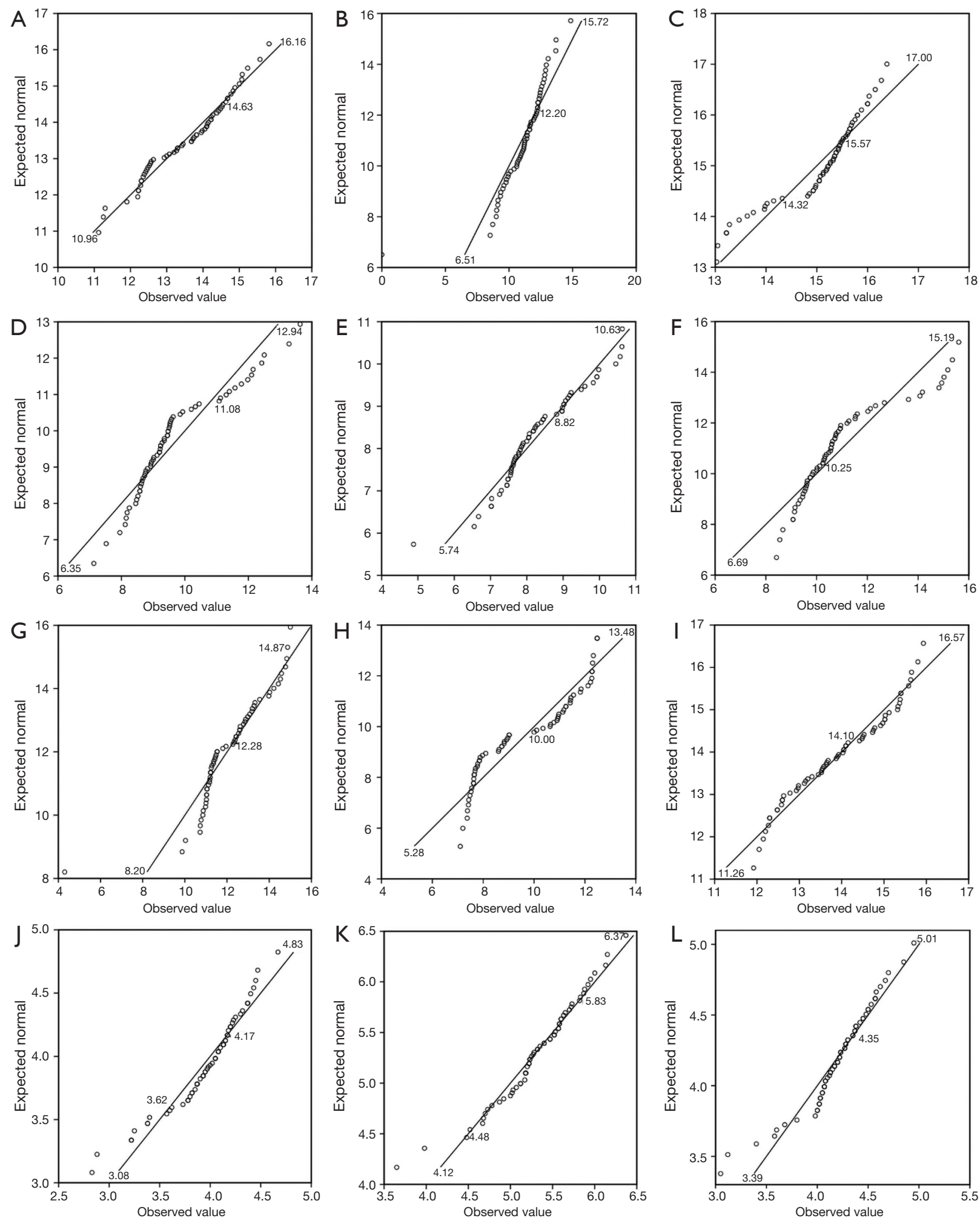

Figure S1 The scatter plots displayed the distribution of mean, minimum and maximum shear wave speed (SWS) and thickness for healthy CET (A,B,C,J), pre-therapy involved CET (D,E,F,K) and post-therapy involved CET (G,H,I,L). The x-axes, y-axes and solid lines represent the observed actual value of SWS and thickness, expected normal values calculated by formula and the theoretical distribution lines. The data are normally distributed as the scatter points are closed to the solid lines. 

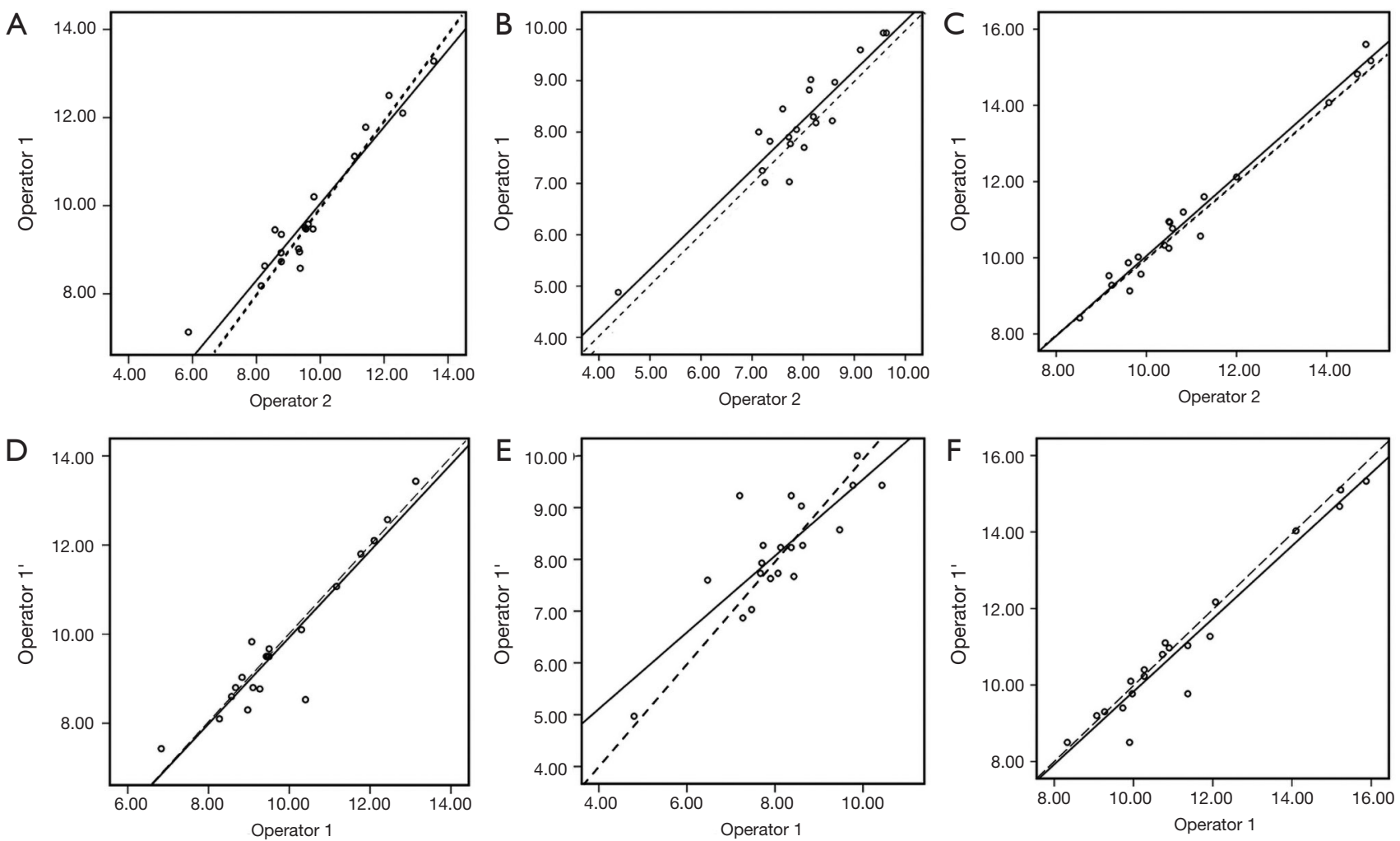

Figure S2 Inter- (A,B,C) and intra-observer (D,E,F) reliability of mean, minimum and maximum shear wave speed (SWS) using the shear wave elastography (SWE). The dashed lines represent 1:1 identity lines and the solid lines represent trend lines of scatter plots. 